Meta

Journal des traducteurs

Translators' Journal

\title{
Le chauffage solaire domestique
}

\section{Lucie Goyette}

Volume 28, numéro 3, septembre 1983

URI : https://id.erudit.org/iderudit/002958ar

DOI : https://doi.org/10.7202/002958ar

Aller au sommaire du numéro

Éditeur(s)

Les Presses de l'Université de Montréal

ISSN

0026-0452 (imprimé)

1492-1421 (numérique)

Découvrir la revue

Citer cet article

Goyette, L. (1983). Le chauffage solaire domestique. Meta, 28(3), 283-292.

https://doi.org/10.7202/002958ar

Ce document est protégé par la loi sur le droit d'auteur. L'utilisation des services d'Érudit (y compris la reproduction) est assujettie à sa politique d'utilisation que vous pouvez consulter en ligne.

https://apropos.erudit.org/fr/usagers/politique-dutilisation/ 


\section{LE CHAUFFAGE SOLAIRE DOMESTIQUE}

Avant la crise énergétique de 1973, l'énergie solaire n'était guère étudiée que par quelques laboratoires universitaires disséminés à travers le monde. Aujourd'hui cependant, cette forme d'énergie renouvelable retient de plus en plus l'attention générale. Les gouvernements accordent des crédits pour stimuler la recherche en ce domaine afin de mettre au point une technologie solaire applicable à l'échelle industrielle.

En HÉLIOTECHNIQUE Ou GÉNIE SOLAIRE (solar engineering), science pratique de l'utilisation de l'énergie solaire, on a depuis quelques années exploré plusieurs procédés de conversion de l'énergie solaire qui sont utilisés pour un grand nombre d'applications: le chauffage domestique, la production d'électricité, le traitement des matériaux (réfractaires), la production d'hydrogène, de méthane, etc. (voir tableau A).

Le type de conversion le plus courant est la CONVERSION PHOTOTHERMIQUE (photothermal conversion, solar thermal conversion) qui consiste en une transformation directe* du rayonnement solaire en chaleur. (On parle de conversion directe lorsque le rayonnement solaire est directement transformé en une énergie quelconque (conversion photothermique, conversion photovoltaique) et de conversion indirecte lorsque le rayonnement subit au moins une transformation préalable: conversion thermodynamique, conversion thermochimique, etc.) L'application la plus répandue est le chauffage solaire domestique. La conversion photothermique peut être réalisée par deux procédés différents : l'effet de serre ou la concentration.

L'EFFET DE SERRE OU EFFET SÉLECTIF (greenhouse effect) est mis en œuvre dans le CAPTEUR PLAN (flat-plate collector) (voir figure 1). Il s'agit du phénomène de piégeage de la chaleur causé par la transparence sélective du verre ou du plastique. Ces matériaux sont utilisés comme COUVERTURE ${ }^{1 *}$ (cover plate)

* Les chiffres en indice renvoient à la figure 1. 
parce qu'ils laissent passer le rayonnement solaire incident tout en restant opaques au rayonnement infra-rouge émis par l'absorbeur. L'ABSORBEUR ${ }^{4}$ (absorber) est le siège de la conversion proprement dite. Il absorbe le rayonnement et le convertit en chaleur. Il est généralement constitué d'une plaque noircie et mate. Ces deux caractéristiques favorisent respectivement une grande ABSORPTIVITÉ (absorptivity) et une faible ÉMISSIVITÉ (emissivity) de l'absorbeur. L'utilisation récente de la SURFACE SÉLECTIVE ou REVÊTEMENT SÉLECTIF ${ }^{5}$ (selective surface, selective radiation coating) permet d'optimiser ces deux propriétés de sorte que l'absorbeur se rapproche du coRPS NOIR (blackbody) idéal dont l'absorptivité est égale à 1 et l'émissivité à 0 . Le REVÊTEMENT ANTIREFLET ${ }^{7}$ (antireflection coating) réduit la perte optique par réflexion sur la couverture. Enfin, pour limiter les pertes dues au rayonnement et à la convection, on peut ajouter une STRUCTURE CELLULAIRE ANTIRAYONNANTE ${ }^{8}$ (cellular structure, honeycomb structure) entre la couverture et l'absorbeur.

Le CHAUFFAGE SOlaIRE (solar heating) constitue l'utilisation la plus répandue du capteur plan. Toute installation de chauffage solaire comporte quatre fonctions: la captation, le transfert, le stockage et la distribution. On distingue deux types de systèmes de chauffage solaire: le système actif et le système passif.

Le SYSTÈME SOLAIRE ACTIF (active solar system, indirect solar system) met en jeu des machines ou des équipements particuliers qui assurent à eux seuls le contrôle thermique de l'habitat. Dans le système actif, chacune des quatre fonctions mentionnées ci-dessus est accomplie par des équipements spécifiques (voir tableau B). Selon le type de fluide caloporteur utilisé, on distingue le système à air et le système à eau.

Dans le système à air, la CAPTATION (collection) est assurée par des CAPTEURS À AIR Ou INSOLATEURS À LAME D'AIR (air collector, air heater). Un ventilateur souffle l'air réchauffé devant et derrière l'absorbeur. Cet air est ensuite acheminé vers une CUVE D'ACCUMULATION (storage tank) par de larges tuyaux entourés d'un isolant. Le sTockaGe (storage) d'un système à air est le plus souvent assuré par un LIT DE ROCHES (rock bed) (voir figure 2). L'air chaud provenant des capteurs se propage dans l'AcCUMULATEUR ì PIERRES (rock bin) auquel il cède de la chaleur. Lorsqu' on a besoin de cette chaleur emmagasinée, un ventilateur envoie un flux d'air frais, provenant souvent de la direction opposée, ce qui a pour effet d'évacuer la chaleur vers les pièces à chauffer. Dans le stockage à lit de pierres, on utilise souvent le principe de la STRATIFICATION (stratification). Il s'agit de la superposition naturelle de différentes couches ou nappes d'air qui, en raison de leurs différences de température, n'ont pas les mêmes masses volumiques. Ainsi, l'air frais provenant d'une extrémité du lit peut être envoyé vers les capteurs, et l'air chaud provenant de l'autre extrémité vers les pièces de la maison. Une autre forme de stockage est possible par l'utilisation d'un SEL EUTECTIQUe ou MÉLANGE EUTECTIQUe (eutectic salt, phase-changing salt). Ces sels présentent l'avantage de fondre à des températures assez basses (environ $25^{\circ} \mathrm{C}$ ). En fondant, ils absorbent la chaleur provenant des capteurs, puis la libèrent en se cristallisant au contact de l'air frais provenant de la maison. Le mélange eutectique est contenu dans la PLAQUe 


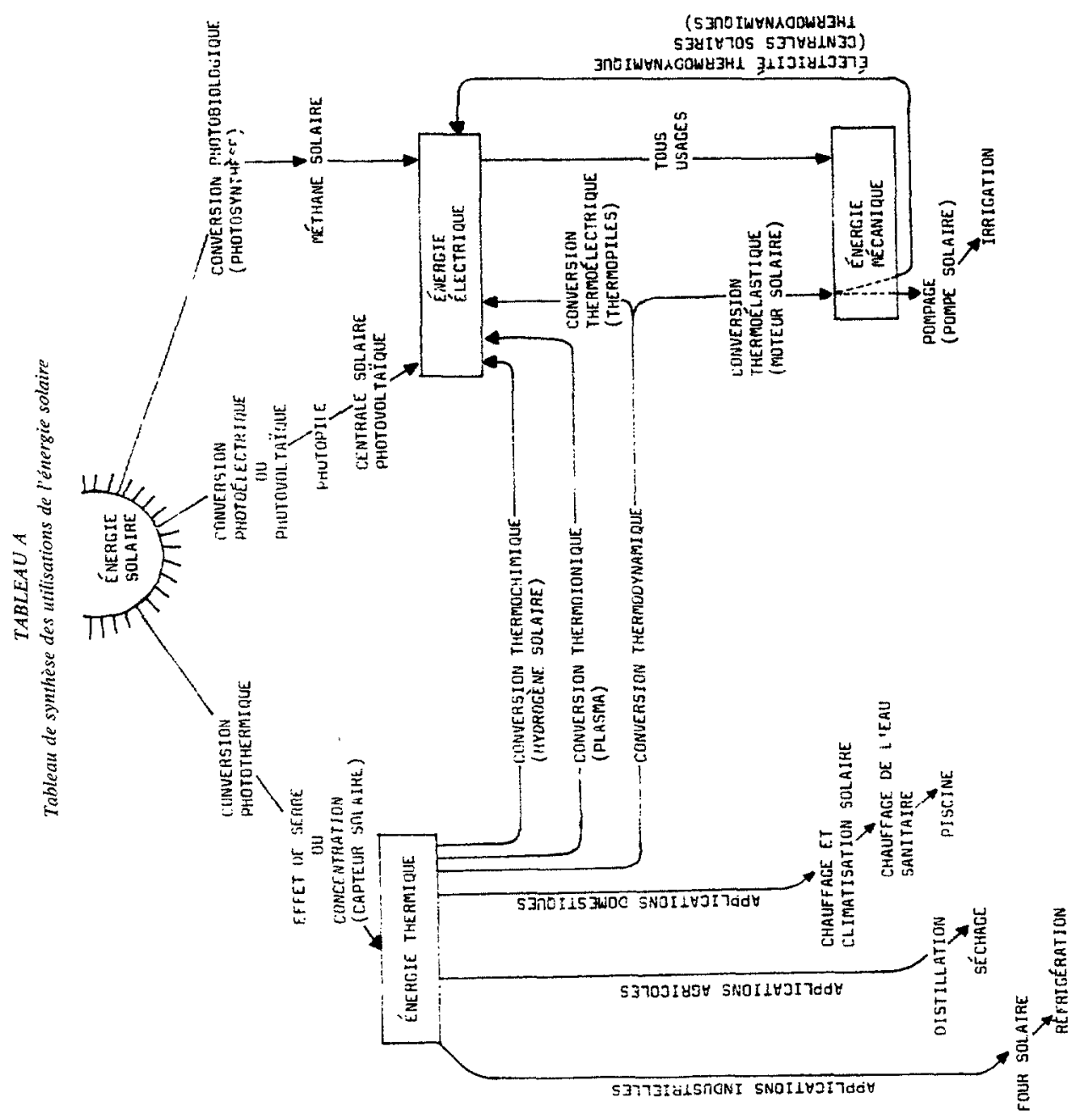


EUTECTIQUe (eutectic tray, eutectic plate). La distribution de la chaleur est assurée par des ventilateurs, des AÉROTHERMES (unit heater) ou une POMPE À CHALEUR (heat pump).

Dans le système à eau, la captation est assurée par le CAPTEUR À EAU ou CAPTEUR À FLUIDE LIQUIDE, encore appelé INSOLATEUR À LAME D'EAU (liquid collector). Le transfert de la chaleur peut s'effectuer par l'intermédiaire d'un CALODUC (head pipe) (voir figure 3) ou d'une POMPE DE CIRCULATION ou CIRCULATEUR (circulation pump) pouvant être montée directement sur les canalisations. L'eau ou le liquide caloporteur est stocké dans un réservoir à eau où l'on peut aussi mettre à profit le phénomène de stratification de différentes couches d'eau. Enfin, la distribution peut être assurée par des radiateurs et une pompe à chaleur.

Par opposition au système actif, le SYSTÈME SOLAIRE PASSIF (passive solar system) n'utilise à peu près pas de machines car il met en jeu des éléments d'architecture. Avec un système passif, toutes les fonctions de captation, de transfert, de stockage et de distribution sont réunies au sein d'un même appareil. Un exemple de système passif est le MUR Trombe (Trombe wall, solar wall) (figure 4) qui utilise le principe de THERMOSIPHON (thermosiphon) suivant lequel un fluide circule sous l'effet des échauffements et des refroidissements qu'il subit le long de son parcours. Parmi les autres systèmes passifs, mentionnons le TOIT CAPTEUR (roof pond) et le MUR D'EAU (water wall).

Tous ces systèmes actifs et passifs ne peuvent cependant pas satisfaire à eux seuls aux besoins en chauffage. On doit les compléter par un CHAUFFAGE D'APPOINT (auxiliary system, back-up system) de type classique.

Il est toutefois possible de concevoir une maison solaire autonome à $100 \%$ en utilisant une architecture qui favorise les apports solaires (ex.: surfaces vitrées au sud) en conjonction avec une ISOLATION VARIABLE (movable insulation) qui réduit les déperditions thermiques pendant la nuit ou les périodes nuageuses.

Comme dans bien d'autres techniques de pointe, les Américains sont les chefs de file en chauffage solaire. Au Québec, où la technologie solaire

\section{TABLEAU B}

Système actîf

\begin{tabular}{|c|c|c|}
\hline Fonction & Système à air & Système à eau \\
\hline $\begin{array}{l}\text { captation } \\
\text { transfert }\end{array}$ & $\begin{array}{l}\text { capteur à air } \\
\text { canalisations } \\
\text { ventilateurs }\end{array}$ & $\begin{array}{l}\text { capteur à eau } \\
\text { canalisations } \\
\text { caloduc } \\
\text { pompe de circulation }\end{array}$ \\
\hline stockage & $\begin{array}{l}\text { accumulateur } \longrightarrow \text { stratification } \\
\text { à pierres } \\
\text { plaques eutectiques }\end{array}$ & réservoir à eau \\
\hline distribution & $\begin{array}{l}\text { ventilateurs } \\
\text { aérothermes } \\
\text { pompe à chaleur }\end{array}$ & $\begin{array}{l}\text { radiateurs } \\
\text { pompe à chaleur }\end{array}$ \\
\hline
\end{tabular}




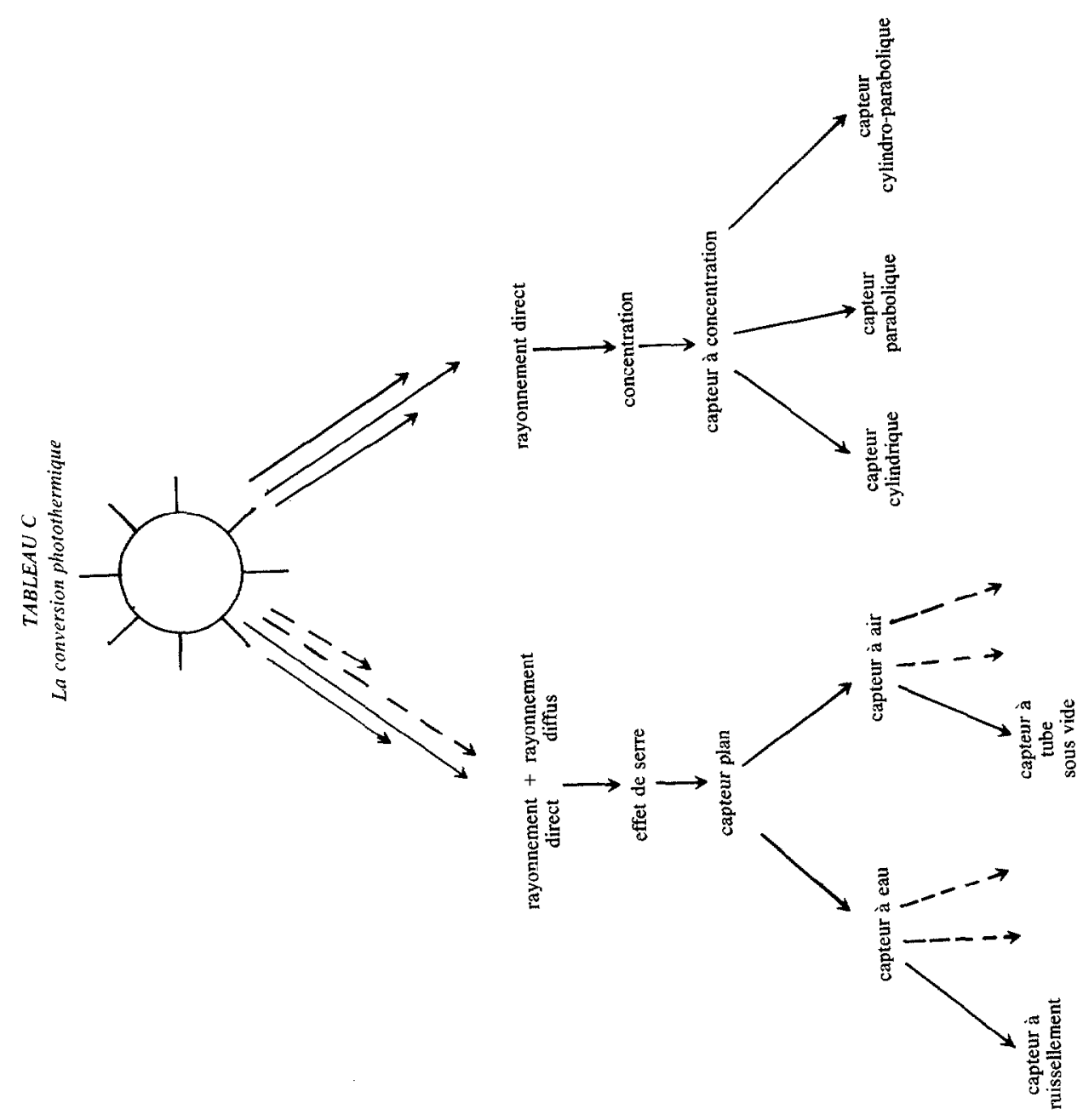


Figure I

Capteur solaire plan à eau

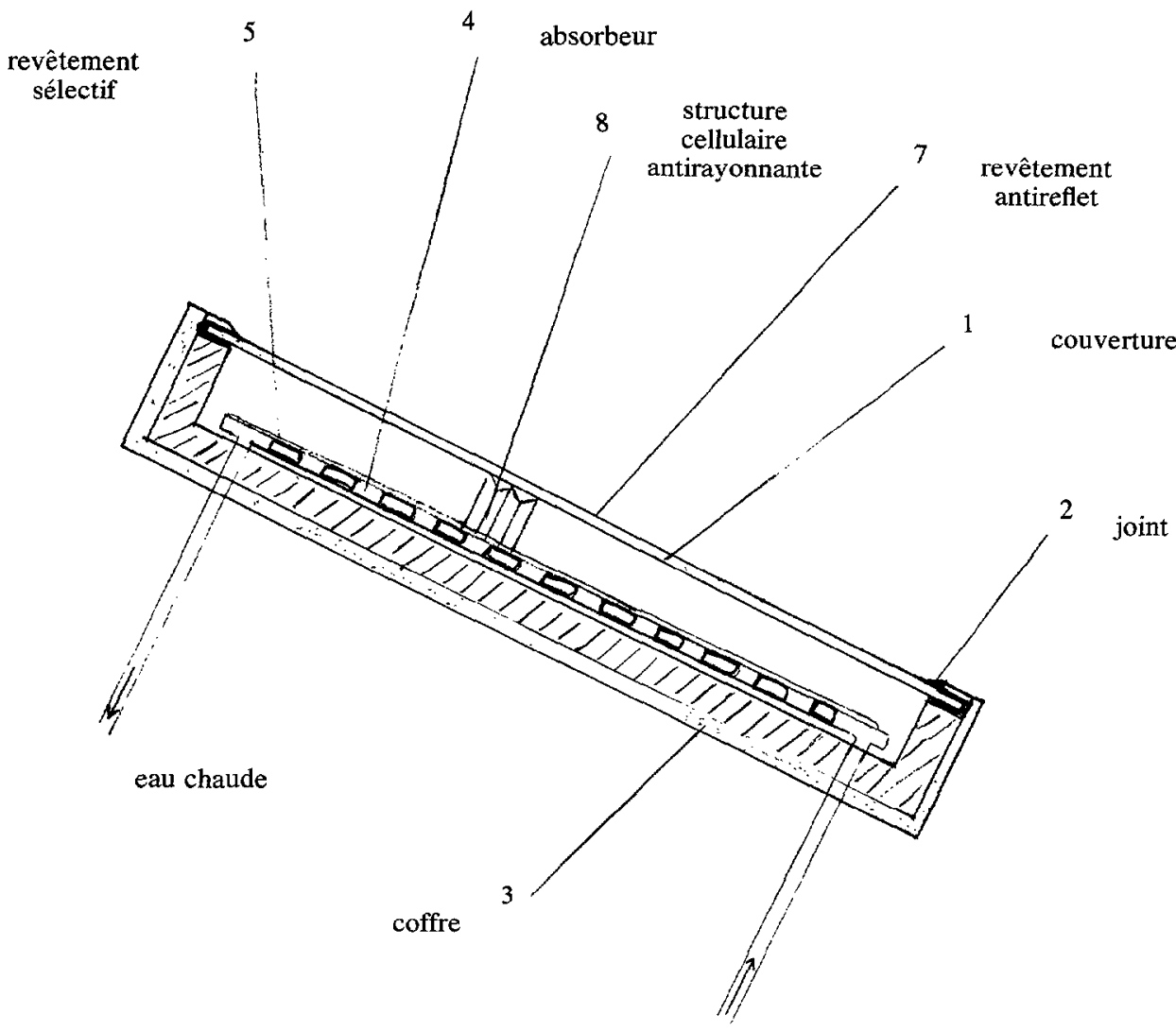

eau froide

commence à s'implanter sérieusement, l'abondance des publications américaines sur ce sujet par rapport au nombre restreint d'écrits français crée un besoin pressant de francisation. Il est essentiel de combler cette lacune et de maintenir la terminologie française à la fine pointe du développement technique.

Petit lexique du chauffage solaire

ABSORBEUR - absorber. Dans un capteur solaire, surface généralement noircie et mate où s'accomplit la conversion thermique du rayonnement solaire ainsi que le transfert de la chaleur au fluide caloporteur.

ABSORBEUR-ÉCHANGEUR - Voir ABSORBEUR.

ABSORPTIVITÉ - absorptivity. Grandeur mesurant l'aptitude d'un corps à absorber la chaleur, définie de façon numérique par l'absorbance. Donnée importante pour l'évaluation du rendement d'un absorbeur. 
ACCÉLÉRATEUR - Voir POMPE DE CIRCULATION.

ACCUMULATEUR À PIERRES - rock storage bin, rock bin. Réservoir de stockage thermique rempli de pierres et utilisé dans les systèmes de chauffage et de climatisation solaire à air.

ACCUMULATION THERMIQUE SOLAIRE - solar heat storage. En chauffage solaire, accumulation de l'énergie solaire convertie en chaleur par le système de captation. Il existe différents types de stockage selon le moyen utilisé : réservoir d'eau, lit de pierres, béton, etc.

AÉROTHERME - unit heater. Appareil destiné au chauffage ou au refroidissement de l'air ou du gaz qui le traverse. L'écoulement de l'air, chauffé ou refroidi à l'aide de l'énergie thermique, est assuré par un ou plusieurs ventilateurs dans un même coffrage ou dans un bloc.

APPORT SOlAIRE - solar gain. Proportion de la charge thermique d'un bâtiment qui est apportée uniquement par le rayonnement solaire réchauffant les parois ou transmis par les fenêtres, à l'exclusion de l'énergie fournie par le système de chauffage solaire actif.

ARCHITECTURE SOLAIRE - solar architecture. Art de concevoir et de construire des bâtiments faisant appel prioritairement au solaire pour satisfaire leurs besoins énergétiques.

BALLON STOCKEUR - Voir CUVE D'ACCUMULATION.

CALAGE - collector tilt angle, collector tilt. Angle formé par le plan de l'absorbeur et le plan horizontal, dont la valeur est déterminée de façon à maximiser le rendement du capteur.

CALODUC - heat pipe. Appareil thermique constitué d'un tube rempli partiellement par un liquide et sa vapeur et entouré d'une mèche en matériau fibreux ou métallique. La chaleur est transférée de la partie chaude à la partie froide du tube par vaporisation de la phase liquide et condensation de la vapeur dans la partie froide.

CAPTAGE - collection. Processus par lequel l'énergie solaire est captée et transformée en chaleur ou en électricité. Le captage de l'énergie sous forme thermique est réalisé soit par effet de serre et piégeage entre deux surfaces, soit par concentration au moyen de miroirs convergents.

CAPTATION - VOIR CAPTAGE.

CAPTEUR À AIR - air type collector, air heater. Capteur solaire thermique utilisant l'air comme fluide caloporteur.

CAPTEUR À CONCENTRATION - concentrating collector, focusing collector. Capteur solaire thermique pourvu de dispositifs réfléchissants ou d'un ensemble de lentilles optiques permettant de concentrer le rayonnement solaire sur une surface d'absorption réduite.

CAPTEUR A EAU - liquid type collector, liquid collector. Capteur solaire thermique plan mis en cuvre avec un fluide caloporteur se présentant sous la forme d'un liquide (habituellement de l'eau).

CAPTEUR À FLUIDE LIQUIDE - Voir CAPTEUR À EAU.

CAPTEUR PLAN - flat-plate collector. Capteur thermique convertissant le rayonnement solaire en chaleur sans concentration préalable. Composé essentiellement d'un absorbeur, d'une couverture transparente et d'une isolation thermique, le capteur plan met en ouvre le phénomène d'effet de serre.

CAPTEUR SOLAIRE - solar collector, solar receiver. Appareil destiné à recevoir le rayonnement solaire et à assurer sa conversion énergétique le plus souvent sous forme thermique.

CAPTEUR THERMIQUE - VOir CAPTEUR SOLAIRE.

CHAUFFAGE D'APPOINT - auxiliary heating, back-up heating. Chauffage dont l'effet s'ajoute, dans les maisons à chauffage solaire partiel, à celui d'un système de chauffage solaire, et dont la souplesse permet de faire face à des variations rapides des besoins de chaleur.

CHAUFFAGE SOLAIRE - solar heating. Conversion du rayonnement solaire en chaleur, généralement pour des besoins domestiques tels que le chauffage des bâtiments et de l'eau sanitaire.

CONVERSION HÉLIOTHERMIQUE - VOir CONVERSION PHOTOTHERMIQUE.

CONVERSION PHOTOTHERMIQUE - photothermal conversion, heliothermal conversion. Processus de conversion qui consiste en une transformation directe du rayonnement solaire en chaleur. Les convertisseurs photothermiques les plus courants sont les capteurs plans et les capteurs à concentration.

CONVERSION THERMIQUE - Voir CONVERSION PHOTOTHERMIQUE.

CORPS NOIR - blackbody. Toute matière qui absorberait de façon idéale la totalité de la lumière qu'elle reçoit.

COUCHE ANTIRÉFLÉCHISSANTE - VOIr REVÊTEMENT ANTIREFLET.

COUCHE ANTIREFLET - Voir REVÊTEMENT ANTIREFLET.

COUCHE SÉLECTIVE - VOIr SURFACE SÉLECTIVE.

COUVERTURE - cover plate, cover. Élément du capteur solaire plan constitué d'une ou de deux plaques de verre ou de plastique transparent et ayant pour fonction de couvrir l'absorbeur de façon à emprisonner l'énergie solaire par effet de serre.

CUVE D'ACCUMULATION - storage tank, storage bin. Dans un système de chauffage solaire, récipient de très grandes dimensions, rempli d'eau ou de pierres, que traverse le fluide caloporteur 
préalablement chauffé par les capteurs solaires. La chaleur cédée par le fluide caloporteur est alors emmagasinée dans la cuve avant d'être distribuée, selon les besoins, dans toutes les pièces de l'habitation.

CUVE DE STOCKAGE - Voir CUVE D'ACCUMULATION.

DEGRÉ-JOUR - degree-day. Différence algébrique, exprimée en degrés Celsius, entre la température moyenne réelle d'un jour donné et une température de référence. Pour une période donnée, on effectue la somme algébrique des «degré-jour" de la période.

EFFET DE SERRE - greenhouse effect. Phénomène de rétention de la chaleur obtenu grâce à la transparence sélective du verre ou du plastique utilisé pour les couvertures de capteurs solaires; ces matériaux laissent passer le rayonnement solaire incident mais sont opaques au rayonnement infrarouge émis par l'absorbeur et emprisonnent ainsi la chaleur.

EFFET SÉLECTIF - VOir EFFET DE SERRE.

ÉMISSIVITÉ - emissivity, Rapport entre le pouvoir émissif d'un absorbeur et celui d'un corps noir de même forme à la même température.

FACTEUR DE CONVERSION - VOir RENDEMENT DE CONVERSION.

FACTEUR D'ÉMISSION - Voir ÉMISSIVITÉ.

GÉNIE SOLAIRE - Voir HÉLIOTECHNIQUE.

HÉLIOTECHNIQUE - solar engineering, solar technology. Science pratique de l'utilisation de l'énergie solaire; étude des procédés et des appareils de conversion de cette énergie.

INCLINAISON - VOir CALAGE.

INSOLATEUR À CHAUFFAGE D'AIR - VOII CAPTEUR À AIR.

INSOLATEUR Á FONCTION THERMIQUE - VOIT CAPTEUR SOLAIRE.

INSOLATEUR À LAME D'AIR - VOir CAPTEUR À AIR.

INSOLATEUR CONCENTRATEUR - VOIr CAPTEUR Á CONCENTRATION.

INSOLATEUR PLAN - VOir CAPTEUR PLAN.

ISOLATION VARIABLE - movable insulation. Dispositif permettant de réduire les pertes thermiques d'une maison pendant la nuit ou les périodes nuageuses et de laisser entrer les apports thermiques au cours des périodes ensoleillées (ex.: volets, rideaux isolants, volets isolants, Beadwall ${ }^{\mathrm{R}}$.

MÉLANGE EUTECTIQUE - eutectic salt, phase-changing salt. Mélange fondant ou se solidifiant à une température inférieure au point de fusion de chacun de ses constituants. Les mélanges eutectiques sont utilisés pour le stockage thermique dans les systèmes de chauffage et de climatisation solaire.

MUR CAPTEUR - Voir MUR TROMBE.

MUR D'EAU - water wall, drumwall. Mur faisant office de système solaire passif. Il s'agit d'un mur intérieur composé de cylindres remplis d'eau. Les cylindres, dont les extrémités exposées au soleil sont peintes en noir, servent de capteurs en même temps que de réservoirs de stockage.

MUR TROMBE - Trombe wall, solar wall, thermosiphoning solar collector. Système de chauffage solaire passif constitué par un mur de maçonnerie noir recouvert d'un vitrage et orienté vers le sud; les rayons solaires traversent le vitrage et sont absorbés par le mur. Grâce à des ouvertures pratiquées au haut et au bas de la serre, l'air froid y pénètre par le bas et, après avoir été chauffé, monte vers l'ouverture du haut par thermosiphon pour ensuite se propager dans la pièce à chauffer.

MUR TrOMBE-MICHEL - Voir MUR TROMBE.

NID D'ABEILLES - Voir STRUCTURE EN NID D'ABEILLES.

PLAQUE EUTECTIQUE - storage tray, eutectic plate. Appareil de stockage thermique solaire affectant la forme d'un réservoir plat et qui est rempli d'un mélange eutectique.

POMPE DE CIRCULATION - circulation pump, circulator. Petite pompe électrique utilisée pour faire circuler l'eau dans les petites installations de chauffage solaire à eau et pouvant être montée directement sur les canalisations.

RENDEMENT DE CONVERSION - conversion efficiency, conversion factor. Rapport entre l'énergie fournie par un convertisseur d'énergie solaire (capteur solaire, pile solaire, plante, etc.) et l'énergie nécessaire pour produire cette énergie par le biais d'un dispositif de conversion.

RÉSERVOIR DE STOCKAGE - Voir aCCUMULATEUR THERMIQUE SOLAIRE.

REVETTEMENT ANTIREFLET - antireflection coating. Couche mince d'un matériau transparent dont on revêt la couverture du capteur pour réduire la perte optique par réflexion sur le vitrage et augmenter ainsi le facteur de transmission de la couverture.

REVÊTEMENT SÉLECTIF - VOir SURFACE SÉLECTIVE.

SEL EUTECTIQUE - Voir MÉLANGE EUTECTIQUE.

SOLARISATION - retrofitting, refitting. Installation d'un système de captage solaire sur un bâtiment existant.

STOCKAGE À COURT TERME - Voir STOCKAGE DE COURTE DURÉE. 
STOCKAGE À LIT de CAILlOUX - Voir STOCKAGE À LIT de RoCHES.

STOCKAGE À LIT DE GALETS - Voir STOCKAGE À LIT DE ROCHES.

STOCKAGE À LIT DE ROCHES - rock bed storage, gravel bed storage, pebble bed storage. Mode de stockage de l'énergie solaire utilisant un accumulateur à pierres et généralement employé dans les systèmes à air. L'air chaud provenant des capteurs se propage dans le réservoir, réchauffant ainsi les pierres. Par la suite, un flux d'air frais provenant souvent de la direction contraire évacue la chaleur vers les pièces à chauffer.

STOCKAGE COURT - VOir STOCKAGE DE COURTE DURÉE.

STOCKAGE DE COURTE DURÉE - short term storage. Dans un système de chauffage solaire, installation d'accumulation thermique dont la capacité ne dépasse pas quelques jours. Dans les régions nordiques ce type de stockage ne fournit que 50 à 70 pour cent du chauffage nécessaire. On doit donc lui adjoindre un chauffage d'appoint.

STOCKAGE DE LONGUE DURÉE - long terme storage, interseasonal storage. Dans un système de chauffage solaire, installation d'accumulation thermique pouvant fonctionner suivant un cycle prolongé ou même annuel, de sorte que le système de chauffage peut fonctionner à 100 pour cent avec l'énergie solaire.

STOCKAGE INTERSAISONNIER - VOir STOCKAGE DE LONGUE DURÉE.

STOCKAGE JOURNALIER - VOIT STOCKAGE DE COURTE DURÉE.

STOCKAGE QUOTIDIEN - VOII STOCKAGE DE COURTE DURÉE.

STOCKAGE THERMIQUE SOLAIRE - VOII ACCUMULATION THERMIQUE SOLAIRE.

STRUCTURE ALVÉOLAIRE SÉLECTIVE - cellular structure, honeycomb structure. Série de cellules identiques de forme géométrique simple (cylindres à base circulaire ou hexagonale, plans parallèles, etc.), que l'on dispose entre la couverture et l'absorbeur d'un capteur solaire pour limiter les pertes dues au rayonnement et à la convection.

STRUCTURE ANTI-PERTE - VOir STRUCTURE ALVÉOLAIRE SÉLECTIVE.

STRUCTURE DE FRANCIA - VOII STRUCTURE EN NID D'ABEILles.

STRUCTURE EN NID D'ABEILLES - honeycomb cellular structure, honeycomb. Structure alvéolaire sélective constituée de cylindres à base hexagonale.

STRUCTURE CELlULAIRE ANTIRAYONNANTE - Voir STRUCTURE ALVÉolaIRE SÉleCtIVE.

SURFACE ABSORBANTE - VOIr ABSORBEUR.

SURFACE SÉLECTIVE - selective surface, selective radiation coating. Dans le domaine du génie solaire, surface ayant un pouvoir d'absorption élevé pour le rayonnement solaire visible, mais un pouvoir émissif faible pour le rayonnement infrarouge.

SYSTEME SOLAIRE ACTIF - active solar system, indirect solar system. Système de chauffage et de climatisation solaire qui fait appel à des machines ou des équipements particuliers assurant à eux seuls le contrôle thermique de l'habitat.

SYSTÈME SOLAIRE MÉCANISÉ - VOIr SYSTÈME SOLAIRE ACTIF.

SYSTÈME SOLAIRE NATUREL - VOir SYSTÈME SOLAIRE PASSIF.

SYSTÈME SOLAIRE PASSIF - passive solar system, direct solar system. Système thermique de chauffage et de climatisation solaire qui utilise certains éléments de l'architecture et dont le fonctionnement est autonome. Ces systèmes se distinguent des systèmes actifs par le fait qu'ils ne mettent pas en jeu de machines ni d'autres équipements particuliers.

TOIT CAPTEUR - roof pond. Système de chauffage et de climatisation solaire passif où l'absorption et le stockage de la chaleur sont assurés par un ensemble de sacs plastiques noirs remplis d'eau. Ces sacs, montés sur le toit, sont recouverts d'un film plastique transparent. La chaleur est transmise directement vers l'intérieur de la maison.

TUBE EN BANDE - tube-in-plate absorber, tube-in-sheet absorber, tabe-in-strip. Plaque absorbante en aluminium ou en cuivre noirci, permettant une circulation d'eau intégrée grâce aux canaux fabriqués à même la plaque par le procédé Roll-Bond, par laminage à chaud et à froid.

TUBE EN NAPPE - VOir TUBE EN BANDE.

VIDANGE - drain down, self-draining system. Dans les capteurs à fluide liquide, dispositif permettant la vidange automatique des eaux de circulation quand l'installation ne capte plus de chaleur. Grâce à ce système, la canalisation ne risque pas d'être endommagée en cas de gel.

TEMPÉRATURE D'ÉQUILIBRE - stagnation temperature, equilibrium temperature. Température atteinte par un fluide caloporteur lorsque, dans un capteur solaire plan, les pertes thermiques sont égales aux apports thermiques. A cette température d'équilibre, le rendement du capteur solaire est nul.

TEMPÉRATURE D'EXPLOITATION - Voir TEMPÉRATURE DE FONCTIONNEMEMT.

TEMPÉRATURE DE FONCTIONNEMENT - operating temperature. Dans un capteur solaire, température de sortie minimale que l'on peut espérer atteindre dans des conditions dites normalisées. La température de fonctionnement est étroitement reliée au débit du fluide caloporteur. Nor- 
malement, plus le débit est élevé, plus la température de fonctionnement descend, ce qui augmente le rendement du capteur.

TEMPÉRATURE LIMITE - Voir TEMPÉRATURE D'ÉQUILIBRE.

LuCiE GoyetTe 